\title{
Ultrafast plasmon thermalization in epitaxial graphene probed by time-resolved THz spectroscopy
}

Vaisakh C. Paingad ${ }^{1}$, Jan Kunc ${ }^{2}$, Martin Rejhon ${ }^{2}$, Ivan Rychetský ${ }^{1}$, Ivan Mohelský3, Milan Orlita ${ }^{2,3}$, and Petr Kužel ${ }^{1 *}$

${ }^{1}$ Institute of Physics of the Czech Academy of Sciences, Na Slovance 2

18221 Prague 8, Czech Republic

${ }^{2}$ Faculty of Mathematics and Physics, Charles University, Ke Karlovu 3, 12116 Prague 2, Czech Republic

${ }^{3}$ Laboratoire National des Champs Magnétiques Intenses, Université Grenoble Alpes, CNRSUPS-INSA-EMFL, 25 rue des Martyrs, Boîte Postale 166, 38042 Grenoble Cedex 9, France *kuzelp@fzu.cz

Keywords: epitaxial graphene, localized plasmon, terahertz, sheet conductivity, ultrafast, Fermi level, carrier cooling

\begin{abstract}
The control of carrier transport by electrical, chemical or optical Fermi level tuning is central to graphene electronics. We apply optical pump—-terahertz $(\mathrm{THz})$ probe spectroscopy to investigate ultrafast sheet conductivity dynamics in various epitaxially grown graphene layers representing a large variety of carbon allotropes, including $\mathrm{H}_{2}$ intercalated films. Our graphene layers display a prominent plasmonic response connected with induced $\mathrm{THz}$ transparency spectra on ultrashort timescale. It is generally believed that the plasmonic excitations appear due to wrinkles, and substrate terraces that bring about natural confinement potentials. We show that these potentials act within micrometer-sized domains with essentially isotropic character. The measured ultrafast dynamics are entirely controlled by the quasi-Fermi level of laser-excited carriers through their temperature. The photocarriers undergo a disorderenabled super-collision cooling process with an initial picosecond transfer of the optically deposited heat to the lattice followed by a sub-nanosecond relaxation governed by the lattice cooling. We describe the transient spectra by a two-temperature Drude-Lorentz model revealing the ultrafast evolution of the carrier temperature and chemical potential and providing
\end{abstract}


crucial material parameters such as Fermi energy, carrier mobility, carrier confinement length, and disorder mean free path.

\section{Introduction}

Graphene layers show a great potential for various applications in nanoelectronics owing to a highly efficient charge carrier transport within the two-dimensional sheets and owing to the tunability of the Fermi level by a voltage or chemical control. Such properties make the usage of graphene suitable for incorporating it into other active or passive optical devices, for example for the development of field effect transistors, ${ }^{[1]}$ detectors of far infrared radiation, ${ }^{[2]}$ optical sensing ${ }^{[3]}$ or, e.g., for graphene-based electrode applications. ${ }^{[4,5]}$ Very strong nonlinear properties of graphene have been also reported including harmonic generation in the $\mathrm{THz}$ spectral range ${ }^{[6]}$ and saturable absorption. ${ }^{[7]}$ Plasmonic properties of graphene have been extensively studied. Surface plasmons on graphene were modelled ${ }^{[8]}$ and can be optically launched ${ }^{[9]}$ with a number of applications through the terahertz and infrared range; ${ }^{[10,11]}$ recently, tunable localized plasmonic behavior of patterned graphene and graphene ribbons have been demonstrated and studied. ${ }^{[12]}$

The two candidates for transfer from fundamental research to the industry are chemical vapor deposition (CVD) graphene ${ }^{[4,13]}$ and epitaxial graphene. ${ }^{[14,15]}$ Their electronic quality is comparable; however, epitaxial graphene grows directly on an insulating substrate, and thus, no complications with contamination and structural defects arise. Epitaxial graphene on silicon carbide $(\mathrm{SiC})$ also provides a unique tool to prepare and study several carbon allotropes which are inherent to the growth on SiC. These carbon allotropes are buffer layer (BL), single-layer graphene (SLG), quasi free-standing single layer graphene (QFSLG), quasi free-standing bilayer graphene (QFBLG), and multi-layer epitaxial graphene (MEG). 
The Si-face of SiC can host four allotropes (BL, SLG, QFSLG, QFBLG), ${ }^{[16]}$ and the C-face, where graphene grows faster, hosts the MEG. ${ }^{[17]}$ The BL is a carbon layer partially $\mathrm{sp}^{3}$ bonded to the $\mathrm{SiC}$ substrate. The SLG consists of a BL and an additional $\mathrm{sp}^{2}$ bonded carbon layer; the $\mathrm{sp}^{2}$ bonded carbons show the pristine graphene properties. After intercalation in hydrogen, ${ }^{[18]}$ the $\mathrm{BL}$ bonding to the substrate vanishes; thus, the $\mathrm{BL}$ turns into a new $\mathrm{sp}^{2}$ bonded carbon layer, graphene. As a result, the intercalated BL turns into QFSLG, and SLG turns into QFBLG. ${ }^{[16]}$ Since BL, single, and bilayer graphene have different electronic structures, and SLG and QFSLG experience different scattering mechanisms ${ }^{[19,20]}$ the graphene on SiC provides several routes in employing strong light-matter interaction in the far-infrared and terahertz spectral range. The allotropes' variability is vital, and their further understanding is necessary. This concerns especially the viability of $\mathrm{THz}$ devices with as-grown graphene, and the influence of buffer layer and substrate etching on device performance.

Terahertz (THz) spectroscopy is a powerful method to study the charge carrier transport in nanomaterials since it inherently involves contactless probing of the conductivity or photoconductivity by using broadband freely propagating electromagnetic pulses in the farinfrared range. ${ }^{[21,22]}$ Its usefulness for ultrafast carrier transport assessment has been abundantly demonstrated in variously prepared single or multi-layer graphene, ${ }^{[22-24]}$ graphene oxide ${ }^{[25,26]}$ and similar $2 \mathrm{D}$ systems. ${ }^{[27]}$

In the optical spectral range, the absorption of photons in graphene is achieved via vertical inter-band transitions leading to the formation of electrons and holes with high kinetic energy. Within the sub-picosecond time range these carriers redistribute in the wave vector-energy space to obey the Fermi-Dirac distribution characterized by hot-carrier temperature $T_{c}$, which can largely exceed the lattice temperature $T_{l}$. Such electrons may produce hot carrier nonlinearities and will exhibit a relaxation to the lattice temperature through electron-phonon coupling: such dynamics can be conveniently followed by ultrashort broadband THz pulses. In 
the $\mathrm{THz}$ spectral regime, the conductivity of graphene sheets is governed by intra-band absorption, which is usually well-described by the Drude model. In some cases, the carriers may be localized inside artificially prepared epitaxial graphene stripes ${ }^{[12,28]}$ or due to terracelike structure of the underlying $\mathrm{SiC}$ substrate; ${ }^{[29]}$ their plasmon-like response then can be described by a Lorentz damped harmonic oscillator function. ${ }^{[28]}$ The $\mathrm{THz}$ bandwidth thus matches the relevant spectral range for the investigation of the intraband transport processes in graphene.

Here, we present a study of the time-resolved response of all epitaxial graphene (epigraphene) allotropes in the $\mathrm{THz}$ and multi-THz spectral range. We demonstrate that the heated carrier distribution on a picosecond time-scale after optical excitation controls the plasmonic resonance via a temperature-dependent Fermi level.

\section{Experimental results}

The list of samples and their nominal properties are summarized in Table 1. The obtained spectra of the steady-state sheet conductivity are presented in Figure 1 For QFSLG and MEG the observed sheet conductivity shows fingerprints of the localized response: increasing real part and negative imaginary part of the conductivity at low frequencies and a broad conductivity peak observed in the THz spectral range. These features demonstrate that there is a high concentration of mobile carriers available close to the Fermi level in the graphene layers in the ground state in these samples; the particular form of the spectra will be discussed along with the transient photoconductivity data. The spectra of SLG and QFBLG do not show any significant deviation from a Drude-like behavior, while sample BL does not exhibit any measurable conductivity. Much fewer mobile charges are thus available in the ground state of these samples compared to samples MEG and QFSLG. 
Table 1. Growth conditions and nominal characteristics of studied samples.

\begin{tabular}{|c|c|c|c|c|c|}
\hline Sample & $\begin{array}{c}\text { Growth } \\
\text { temperature/time }\end{array}$ & $\begin{array}{c}H_{2} \text { intercalation } \\
\left(1120-500^{\circ} \mathrm{C}\right)\end{array}$ & $\begin{array}{c}\text { Substrate } \\
\text { side }\end{array}$ & $\begin{array}{c}\text { No. of } \\
\text { graphene layers }\end{array}$ & Graphene \\
\hline MEG & $1670^{\circ} \mathrm{C} / 5 \mathrm{~min}$ & Yes & C-side & inhomogeneous & $\begin{array}{c}\text { multi-layer epitaxial } \\
\text { graphene }\end{array}$ \\
\hline SLG & $1730^{\circ} \mathrm{C} / 5 \mathrm{~min}$ & No & Si-side & $1+$ buffer layer & single layer graphene \\
\hline QFBLG & $1730^{\circ} \mathrm{C} / 5 \mathrm{~min}$ & Yes (from SLG) & Si-side & & quasi free-standing \\
bilayer graphene
\end{tabular}

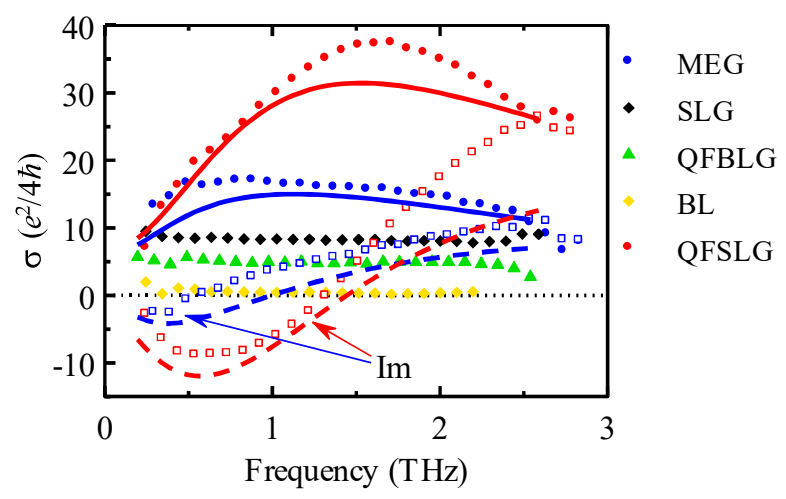

Figure 1. Steady-state spectra of the complex sheet conductivity of graphene layers. Closed symbols: real part; open symbols: imaginary part. Only real parts are shown for samples $S L G, Q F B L G$ and BL. The blue and red lines correspond the global fit for samples MEG and QFSLG, respectively, which includes also all the transient spectra and is described in Sec. 4.

In Figure 2(a) we show examples of the pump probe scans for all the samples using a relatively high incident pump fluence of $\mathrm{F}=0.16 \mathrm{~mJ} \mathrm{~cm}^{-2}$ (i.e., absorbed photon fluence was $8 \times$ $10^{12}$ photons $/ \mathrm{cm}^{2}$ in graphene layers). The onset of the signal just after $\tau_{p}=0$ ps differs in sign for samples MEG, QFSLG and SLG from QFBLG and BL; later on, we will demonstrate that these positive and negative contributions have quite different origin and spectral shape. The positive rapidly decaying signal observed for MEG, QFSLG and SLG corresponds to an optically induced transparency of the graphene layer in the $\mathrm{THz}$ range (i.e., to essentially 
negative $\mathrm{THz}$ differential conductivity). Another rise of the signal with the positive sign is observed at $9 \mathrm{ps}$; it is due to the photoexcitation of the graphene layer by a fraction $(\sim 20 \%)$ of the pump pulse partially reflected at the rear surface of the substrate (etalon photoexcitation). This event gives rise to quite an intense differential signal although only a fraction of the incident pump pulse fluence is available here; we can then infer that the induced transparency part of the signal scales nonlinearly with the pump fluence. SLG exhibits induced transparency dynamics on a significantly shorter timescale than MEG and QFSLG: as illustrated in Figure 2, the observed peak in the positive pump-probe signal occurs within one picosecond, which is at the limit of the time resolution of THz spectroscopy. Similar signal of this type, but very weak, is also observed in QFBLG: a small etalon peak is visible near 10 ps and a tiny shoulder near $1 \mathrm{ps}$ in the initial signal build up can be distinguished after a careful examination of the pumpprobe scan.
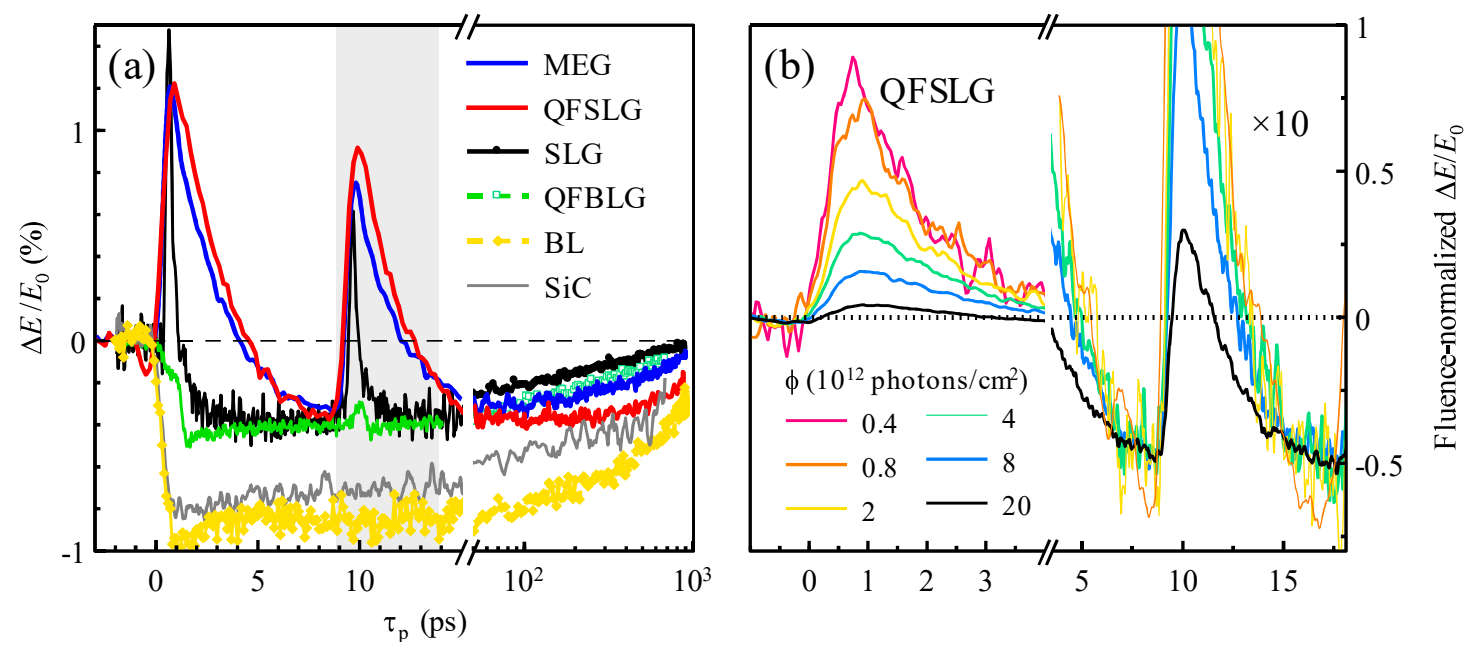

Figure 2. Pump-probe scans of graphene samples measured at the maximum of the transient THz wave form $\Delta E_{\max }$. (a) Summary of all the samples including bare substrate for incident photon fluence $F=$ $0.16 \mathrm{~mJ} / \mathrm{cm}^{2}$ (corresponding to absorbed photon fluence of $\phi=8 \times 10^{12}$ photons $/ \mathrm{cm}^{2}$ in graphene layer). The grey zone at $9-14$ ps contains a signal due to the etalon photoexcitation. Note that the righthand-side part of the plot showing long-lived negative signals has a log scale on the horizontal axis. (b) Fluence-normalized pump-probe signal as a function of the absorbed pump fluence $\phi$ for QFSLG. Note, however, that the negative contribution to the photoconductivity of QFSLG (observed near the pump-probe delays of 8 and $17 \mathrm{ps}$ ) scales linearly with $\phi$. 
In general, in our samples, the negative $\Delta E$ signal scales linearly with the pump fluence while the positive signal scales non-linearly; this is demonstrated in Figure 2(b) for QFSLG. It suggests that the negative signal is simply proportional to the concentration of photoexcited carriers while the positive signal is not, and some nonlinear effects prevail in this photoconductivity contribution.

A measurable negative pump-probe signal, corresponding to a pump induced absorption, is observed in the bare substrate. As demonstrated in Supporting material (Sec. 6), it has a Drude like spectral shape and, in our experimental conditions, it scales linearly with the pump pulse fluence. The linear scaling excludes a two-photon absorption origin, consequently, it should be related to the photoexcitation of electrons from impurity or surface levels to the conduction band in SiC. The pump-probe signal in BL is also negative over the whole measured range of the pump-probe delays, it does not feature any induced transparency peak and it is similar both in magnitude and in shape to the signal observed in the bare substrate. It scales perfectly linearly with the incident pump fluence and it is likely that a part of it comes from the substrate itself. The negative photoconductivity signal in other graphene samples scales also linearly with the pump fluence as demonstrated in Figure 2(b) in the case of QFSLG; however, its magnitude is significantly reduced compared to the one detected in the substrate, see Figure 2(a). Thus, a part of this signal should come either from the layer itself or should be related to the interaction between the film and the substrate.

At times 50 ps or more after photoexcitation all the samples exhibit a negative $\Delta E$ (induced THz absorption), which decays within a few hundreds of picoseconds. 


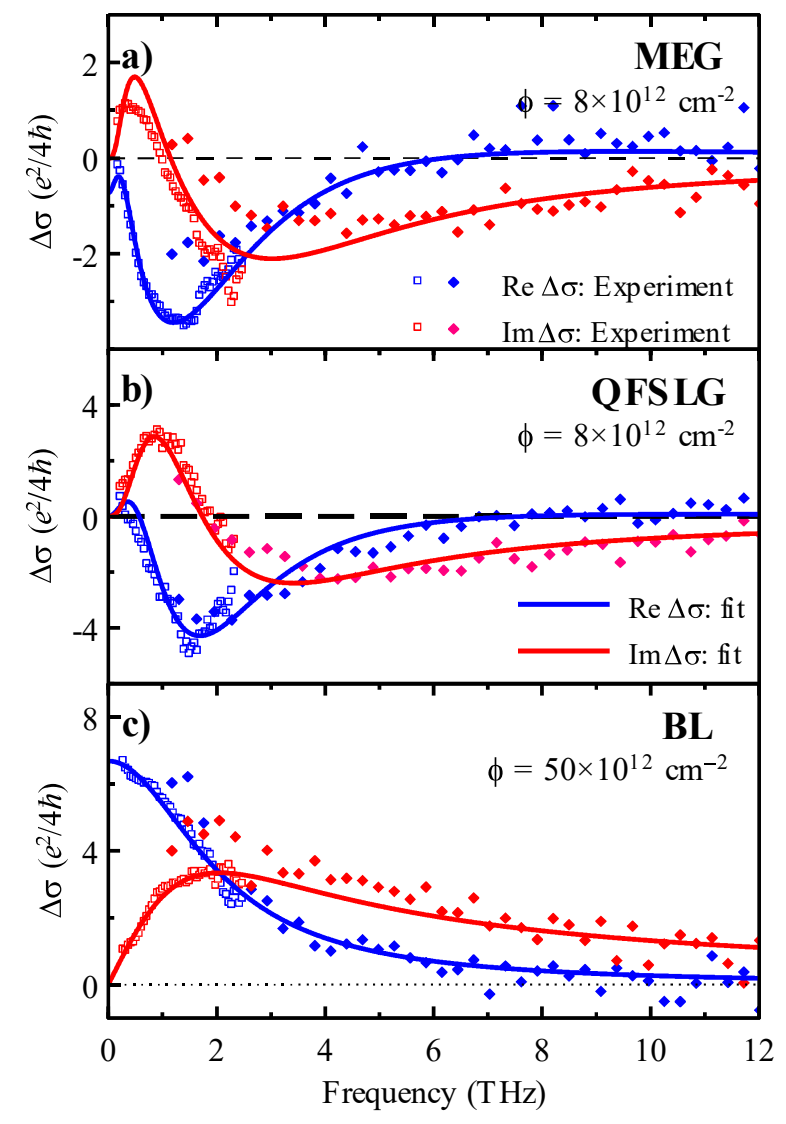

Figure 3. Transient sheet conductivity spectra of samples MEG (a), QFSLG (b) and BL (c). The legends are common for all three plots. Open symbols were obtained using the standard THz setup and closed symbols were obtained in air-based multi-THz experiment. The solid lines represent fits with either the two-temperature model $\triangle \sigma_{\text {dif }}$ (MEG and QFSLG) or the Drude model $\triangle \sigma_{D}(B L)$. Pump-probe delay: 1 ps. Absorbed photon fluences in the graphene layers are indicated in the plots; note that the absolute photoconductivity in BL is higher since the sample was submitted to a significantly higher pump fluence in order to obtain a better SNR.

In Figure 3, we show examples of the transient spectra 1 ps after photoexcitation for samples MEG, QFSLG and BL obtained by both THz and multi-THz experiment. The SNR for the multi-THz setup is significantly worse than for the standard $\mathrm{THz}$ setup, nevertheless the $\mathrm{THz}$ and multi-THz data are in a semi-quantitative agreement and provide an interesting qualitative view into the processes of the charge carrier transport. The samples MEG and QFSLG show a resonant type of response with negative non-monotonous real part of the transient conductivity and this kind of spectra is observed for time delay up to 3 ps where the differential pump-probe signal is positive (Figure 2). On the other hand, the transient signal in sample BL has the usual Drude-like character (with positive decreasing real conductivity). It should be also pointed out 
that, as we verified experimentally, the photoconductivity spectra in these samples are isotropic. In other words, they do not change when the orientation of the terraces on SiC surface is varied with respect to the THz pulse polarization. Namely, for samples MEG and QFSLG the Lorentz-like character of the response and its resonant frequency and damping are preserved. Sample SLG exhibits a peak essentially shorter than the THz pulse length. It has been pointed out than in such a case the leading edge of the $\mathrm{THz}$ pulse probes the sample in a different state than the trailing edge and the spectrally resolved $\mathrm{THz}$ signal may exhibit artifacts. ${ }^{[30,31]}$ In other words, the transient spectra then inherently contain frequency mixing features due sub-picosecond evolution of the probed state. We measured a transient $\mathrm{THz}$ spectrum of this sample for a pump-probe delay "near" the peak value (see Supporting material) and it shows a resonant type response; however, due to the above-mentioned phenomena, we do not interpret this spectrum quantitatively. For a delay of 5 ps after photoexcitation the sample exhibits a spectrum approaching the Drude-like response (see Supporting material, Fig. S3). QFBLG also shows a rapid decay of the positive pump-probe signal, posing a challenge similar to that in SLG. Also, the interesting positive contribution is buried in the Drude-like negative dynamics probably because of the low coverage of QFBLG on the substrate. Due to these factors, we did not analyze the QFBLG in detail.

Based on this analysis, we studied the transient spectra of MEG and QFSLG in detail as a function of the pump fluence and pump-probe delay.

\section{Theoretical model}

When excited with an above-bandgap optical pulse, classical semiconductors usually show an increase in the real conductivity, i.e., positive photoconductivity related to the increase of carrier concentration in the conduction band. Optically excited graphene, on the other hand, is 
known to feature positive or negative sheet photoconductivity $\Delta \sigma_{\text {Real }}$ depending on the material parameters. ${ }^{[32-36]}$

Negative sheet photoconductivity is a rarely observed phenomenon, but it is an interesting one, since it always must be related to a decrease of the conductivity upon photoexcitation with respect to the ground state. Many reports account for this polarity flip with the help of the Drude formalism. ${ }^{[34,35,37]}$ In this picture, the optical excitation increases the carrier density and temperature, altering the conductivity parameters such as the Drude weight and scattering rate. Tuning of these two parameters spawns the bipolar behavior in graphene photoconductivity. The polarity of $\Delta \sigma_{\text {Real }}$ then depends on the sample parameters such as Fermi energy, excitation density, carrier temperature, etc.

\subsection{Drude response}

In our experiments we observed a positive photoconductivity in samples SLG, QFBLG, BL and also in samples QFSLG and MEG at long pump-probe delay times (Figure 2). As it was shown in Figure 3(c) for BL and as it will be also demonstrated later for other samples; this contribution has a Drude-like spectral shape characteristic for free charge carriers described by:

$$
\sigma_{\mathrm{D}}(\omega)=\frac{\sigma_{\mathrm{D}}^{0}}{1-i \omega \tau}
$$

This term could originate in unconfined carriers in the graphene film (denoted by $\sigma_{D}^{G}$ in the following) and in the free carriers in the leftover buffer or other non-graphene species (denoted by $\left.\sigma_{D}^{B}\right)$; a part of the signal also comes from the substrate $\left(\sigma_{\mathrm{SiC}}\right)$, as demonstrated in Figure 2 and in Supporting material (Sec. 6). The sheet photoconductivity amplitude $\sigma_{\mathrm{D}}^{0}$ is proportional to the free carrier density $n$ in a 3D system with parabolic dispersion or to its square root $\sqrt{n}$ in the case of a 2D system (graphene) with linear band structure. Usually, the Drude weight in 
graphene is introduced as follows: $D=\pi \sigma_{\mathrm{D}}^{0} / \tau$. For monolayer graphene, $D$ strongly depends on the chemical potential (quasi-Fermi level) $\mu$, which, in turn, is a function of the carrier concentration, and on the carrier temperature $T_{c}:^{[37]}$

$$
D\left(T_{c}\right)=\frac{2 e^{2}}{\hbar^{2}} k_{B} T_{C} \log \left[2 \cosh \left(\frac{\mu\left(T_{c}\right)}{2 k_{B} T_{c}}\right)\right] .
$$

\subsection{Plasmonic response}

Interestingly, the graphene grown on the $\mathrm{SiC}$ substrate is known to exhibit the carrier localization features. ${ }^{[29]}$ The terraces in $\mathrm{SiC}$ substrate and inherent wrinkles in epitaxial graphene provide the natural confinement potentials for the carriers and such potentials enable the coupling of $\mathrm{THz}$ radiation to localized plasmons ${ }^{[38,39]}$, which is impossible in a perfectly flat homogenous graphene. Similar effects have been also observed in artificially prepared graphene stripes. ${ }^{[12,28,40]}$ Such a mechanism transforms a Drude term describing the free carriers into a Lorentz-like response taking into account their resonant plasmonic character. Such a mechanism can be attributed to both steady-state and transient spectra in samples QFSLG and MEG, where the localized dynamics is observed, see. Figure 1 and Figure 3(a, b).

Therefore, we explain the initial THz sheet photoconductivity in QFSLG and MEG (i.e., first few picoseconds featuring the pump-induced transparency in Figure 2) with the help of a differential Lorentz model. The optical excitation increases the density $(n)$ and temperature ( $T_{c}$ ) of charge carriers, which changes the Lorentz parameters such as the oscillator strength (related to the Drude weight $D$ ), plasma frequency $\left(\omega_{0}\right)$, and linewidth $(\Gamma)$. The observed transient sheet conductivity is the difference between the Lorentz conductivity of excited and unexcited graphene:

$$
\Delta \sigma_{\mathrm{dif}, L}=\sigma_{L}\left(n_{e q}+\Delta n, T_{c}>T_{R T}\right)-\sigma_{L}\left(n_{e q}, T_{c}=T_{R T}\right)
$$


Here, $T_{R T}(\approx 300 \mathrm{~K})$ is the equilibrium lattice temperature, $n_{e q}$ is the equilibrium carrier concentration and $\Delta n$ is the concentration of photoexcited carriers. The Lorentz sheet conductivity reads:

$$
\sigma_{L}(\omega)=\frac{D}{\pi}\left(\frac{i \omega}{\omega^{2}-\omega_{0}^{2}+i \Gamma \omega}\right)
$$

The Drude weight $D$ has been defined by Equation (2); this equivalence is also justified in the Supporting material (Sec. 2). The Lorentz parameters such as plasma frequency $\left(\omega_{0}\right)$, and linewidth $(\Gamma)$ are influenced by the Drude weight and chemical potential: ${ }^{[12,28]}$

$$
\begin{gathered}
\omega_{0}^{2}\left(T_{c}\right) \approx \xi \cdot \frac{D\left(T_{c}\right)}{\varepsilon_{0}}, \\
\Gamma\left(T_{c}\right) \equiv 1 / \tau\left(T_{c}\right)=\frac{e v_{F}^{2}}{\eta_{c} \mu\left(T_{c}\right)}+\gamma T_{c} .
\end{gathered}
$$

Here, $\eta_{c}$ denotes the carrier mobility (we use this unusual notation in order to avoid the confusion with the chemical potential $\mu$ ); $\gamma$ is the LA phonon scattering coefficient. ${ }^{[12,28,41,42]}$ The geometrical parameter $\xi$ often arises from the patterning of the graphene layer. For our unpatterned graphene, $\xi$ is related to inhomogeneities in the samples arising from the substrate terraces and frequent sample fragmentation and wrinkling. It can be understood within a 2D core-shell effective medium model, ${ }^{[43]}$ in which perfect graphene regions (core) are surrounded by ribbons with highly decreased conductivity due to wrinkles or discontinuities (shell). As shown in the Supporting material (Sec. 2), such a model yields:

$$
\xi=\frac{w}{d_{G}^{2} \varepsilon_{W}}
$$

where $d_{G}$ is the characteristic size of the perfect graphene region, $w$ is the width of the zone with highly reduced conductivity and $\varepsilon_{W}$ is the background permittivity of this zone. 

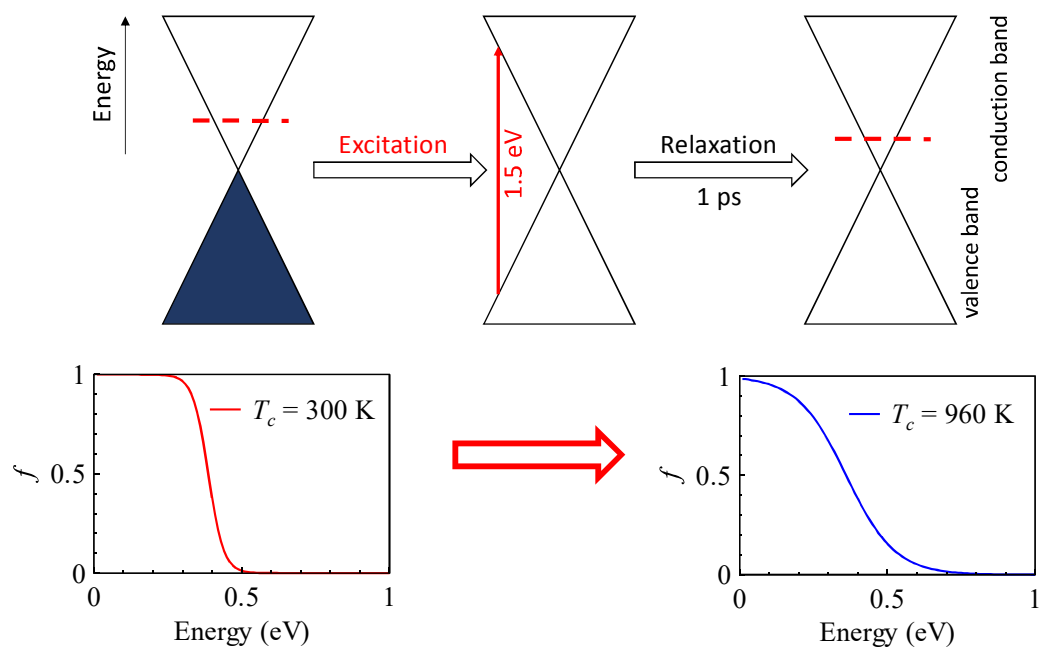

Figure 4. Illustration of the two-temperature model. The optical excitation generates new photocarriers in the epitaxial graphene (here represented as $n$-doped in the ground state) which relax and recombine due to strong scattering and Auger processes. A large fraction of energy dumped to the electronic system by the pump pulse is hence lost to the lattice in this process. Within a few hundreds of femtoseconds, the electronic system relaxes to a warmer carrier distribution with a well-defined temperature $T_{c}>T_{R T}=300 \mathrm{~K}$. The measured $\Delta \sigma_{\text {dif }}$ is the difference in the Lorentz conductivity between the electronic system that is in equilibrium $\left(T_{C}=T_{R T}\right)$ and the one heated due to optical excitation $\left(T_{C}>T_{R T}\right)$. The respective Fermi-Dirac distribution functions are shown in the lower part of the figure.

Sample MEG consists of monolayers or stacks of decoupled monolayers (up to 5 layers) of graphene. Each graphene layer may have somewhat different Fermi energy and carrier mobility. The Fermi energy (and thus the carrier concentration) of $\mathrm{C}$-side grown graphene was reported to increase quite dramatically from the outermost graphene layer towards the innermost one (adjacent to the substrate). ${ }^{[4]}$ Following our description, the equilibrium concentration of carriers determines the magnitude of the observed $\mathrm{THz}$ signal: either directly in $\mathrm{THz}$ steady-state measurements or through their laser-induced heating in the transient experiments. A rigorous addition of individual sheet conductivities is not feasible, since a large number of unknown parameters would be involved, and all of these could not be determined from the experimental results. Hence, we use a simplified approach and treat the MEG system as an "effective layer" with an effective-Fermi energy and carrier mobility; these values then characterize the properties of layers next to the substrate, which provide the prominent $\mathrm{THz}$ 
signal. As a matter of fact, MEG and QFSLG show quite similar pump-probe dynamics and spectral features. Hence, they are both analyzed in the monolayer graphene framework.

\subsection{Plasmonic and carrier cooling}

Within a few tens of femtoseconds after the optical excitation, the electrons and holes acquire a well-defined temperature $T_{c}$ and settle to a Fermi-Dirac distribution in the respective bands. ${ }^{[45]}$ The rapid scattering processes and inter-band Auger recombination ${ }^{[33]}$ merges the electron and hole distributions into a single distribution within a few hundred femtoseconds. Hence it is safe to assume that, within one picosecond after the excitation, the excess carriers recombine $(\Delta n \approx 0)$, and the system is left with a hotter carrier distribution (Figure 4). In our THz experiments, the samples are probed 1 ps after photoexcitation and later; Equation (3) then can be simplified, yielding essentially a two-temperature model:

$$
\Delta \sigma_{\mathrm{dif}, L}=\sigma_{L}\left(n_{e q}, T_{c}>T_{R T}\right)-\sigma_{L}\left(n_{e q}, T_{c}=T_{R T}\right)
$$

Similarly, for a potentially existing population of unconfined carriers, similar two-temperature term can be also written using the Drude response function:

$$
\Delta \sigma_{\mathrm{dif}, D}=\sigma_{D}^{G}\left(n_{e q}, T_{c}>T_{R T}\right)-\sigma_{D}^{G}\left(n_{e q}, T_{c}=T_{R T}\right)
$$

The observed photoconductivity then arises from the heating and cooling of the carrier subsystem, for which the chemical potential $\mu\left(T_{c}\right)$ can be evaluated from the carrier density conservation law: $:^{[46]}$

$$
\frac{1}{2}\left(\frac{T_{F}}{T_{C}}\right)^{2}=F_{1}\left(\frac{\mu}{k_{B} T_{c}}\right)-F_{1}\left(-\frac{\mu}{k_{B} T_{C}}\right) .
$$

Here, $F_{1}$ is the first order Fermi-Dirac integral and $T_{F}=E_{F} / k_{B}$ is the Fermi temperature. One can easily verify that at elevated carrier temperatures, the amplitude of $\sigma_{L}$ drops due to a reduction in the Drude weight accompanied by a broadening and a slight red-shifting of the 
Lorentzian, see Figure 5. The decreased conductivity due to the carrier heating essentially explains the pump-induced $\mathrm{THz}$ transparency observed in samples MEG and QFSLG; it is a highly nonlinear process leading to a nonlinear scaling of the $\mathrm{THz}$ signal as demonstrated in Figure 2(b).

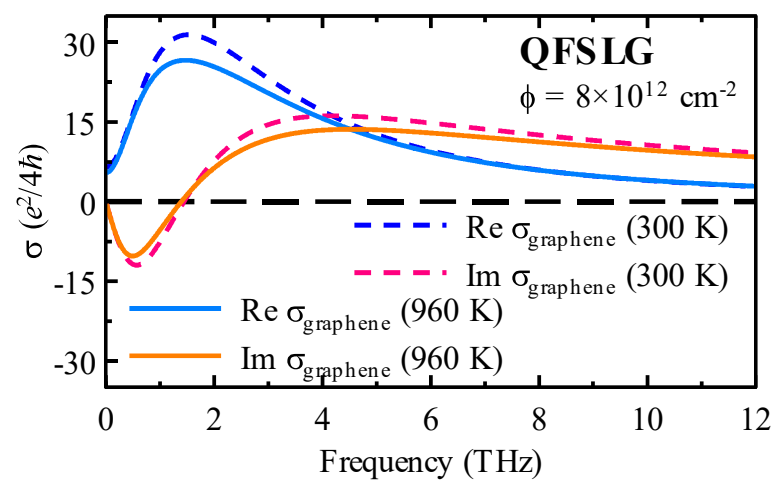

Figure 5. Illustration of the trends of the Graphene conductivity, consisting of the Lorentz part $\sigma_{L}$ and the Drude part $\sigma_{D}^{G}$ entering the two-temperature model, upon an increase in the carrier temperature $T_{C}$; in this plot we used the parameters for sample QFSLG corresponding to the fit in Figure 3(b).

Initial temperature $T_{c}^{0}$ of the electron-hole sub-system shortly after photoexcitation can be estimated from the energy conservation law. ${ }^{[47]}$ For example, for an n-doped graphene sheet excited with an optical pulse depositing an energy $\Delta Q_{\mathrm{op}}$, the energy conservation reads:

$$
U_{e}\left(T_{R T}, n_{e}\right)+\Delta Q_{\mathrm{op}}=U_{e}\left(T_{c}^{0}, n_{e}+\Delta n\right)+U_{h}\left(T_{c}^{0}, \Delta n\right)
$$

The quantity $U_{e / h}$ is the internal energy of the electron/hole sub-system and it is given by the relation, ${ }^{[47]}$

$$
U_{e / h}\left(T_{c}\right)=\frac{4\left(k_{B} T_{c}\right)^{3}}{\pi \hbar^{2} v_{F}^{2}} F_{2}\left(\mu^{e, h} / k_{B} T_{c}\right),
$$

where $F_{2}$ is the second order Fermi-Dirac integral. The equation holds at early times when the absorbed optical energy is not yet substantially dissipated out of the electronic system. However, the carrier-phonon interactions within a picosecond after photoexcitation contribute to a significant reduction in the carrier temperature. Hence one should expect $T_{c}<T_{c}^{0}$ in the $\mathrm{THz}$ photoconductivity response even for the earliest pump-probe measurements $\left(\tau_{p} \geq\right.$ 
$1.5 \mathrm{ps})$. We further assume that, during this first picosecond, the energy is transferred from the electronic sub-system to the lattice without introducing other significant dissipation channels;

this leads to a slight elevation of the lattice temperature $T_{l}^{0}$. Further evolution of $T_{c}\left(\tau_{p}\right)$ follows the super collision cooling (SCC) temperature relaxation process. This process is disorder-enabled and describes enhanced cooling of carriers mediated by their scattering on acoustic phonons. The cooling fulfills equation: ${ }^{[35,48]}$

$$
C_{e} \frac{d T_{c}}{d \tau_{p}}=-\beta\left(T_{c}^{3}-T_{l}^{3}\right)
$$

where $T_{l}\left(>T_{R T}\right)$ is the actual lattice temperature at a given pump-probe delay, $C_{e}=\alpha_{e} T_{c}$ is the electronic specific heat of graphene with $\alpha_{e}=2 \pi k_{B}^{2} E_{F} /\left(3 \hbar^{2} v_{f}^{2}\right)$ and $\beta$ is the super collision cooling coefficient. ${ }^{[12,28,35]}$ The coefficient $\beta$ is related to the Fermi energy $E_{F}$ and the disorder mean free path $l_{\text {dis }}$ as $\beta \propto E_{F} / l_{\text {dis }}$. Following (12) the cooling rate is determined by the ratio $\beta / \alpha_{e}\left(\propto l_{\text {dis }}^{-1}\right)$ and it is independent of $E_{F} \cdot{ }^{[12,35,48]}$ Hence, samples with high degree of disorder would display a fast relaxation dynamics. The graphene lattice is cooled mainly due to the heat transfer to the substrate (through acoustic phonons) and this process occurs on a significantly longer time scale of hundreds of picoseconds; we assume here a phenomenological exponential decay:

$$
T_{l}\left(\tau_{p}\right)=\left[T_{l}^{0}-T_{R T}\right] \cdot \exp \left(-\tau_{p} / \tau_{l}\right)+T_{R T}
$$

\section{Discussion}

The conductivity spectra of MEG and QFSLG show a clear indication of carrier localization (Lorentz term) but a significant free-carrier (Drude-like) background is also observed, namely at longer pump-probe delays. We thus express the measured photoconductivity in these samples as a sum of several terms: 


$$
\Delta \sigma\left(\omega, T_{c}\right)=f \cdot \Delta \sigma_{\mathrm{dif}, L}\left(\omega, T_{c}\right)+(1-f) \cdot \Delta \sigma_{\mathrm{dif}, D}\left(\omega, T_{c}\right)+\Delta \sigma_{\mathrm{SiC}}(\omega)+\Delta \sigma_{D}^{B}(\omega)
$$

Here, the first two right-hand-side terms describe the cooling of confined (Equation 7) and unconfined (Equation 8) carriers ( $f$ is the relative concentration of confined carriers); the third term is the background Drude photoconductivity due to photoexcitation of SiC substrate. Finally, the last term is the Drude photoconductivity (Equation 1) of possibly existing species with parabolic bands. Note that the parameters of the first two terms $\left(T_{c}, D, \Gamma \equiv 1 / \tau\right)$ are connected through the properties of carriers in graphene and that the substrate photoconductivity term does not bring any fitting parameter into the model (the carrier scattering time and the decay of the Drude amplitude were experimentally determined). Since the ground state conductivity of $\mathrm{SiC}$ and of the leftover buffer featuring a band-gap is negligible, the steady-state conductivity can be simply written as

$$
\sigma(\omega)=f \cdot \sigma_{L}\left(\omega, T_{c}=T_{R T}\right)+(1-f) \cdot \sigma_{D}^{G}\left(\omega, T_{c}=T_{R T}\right)
$$

where Equations (14) and (15) are connected through the room temperature properties of carriers. While analyzing the conductivity, we fit the steady-state and photoinduced conductivity spectra simultaneously. The parameters such as Fermi energy $\left(E_{F}\right)$, carrier mobility $\left(\eta_{c}\right)$, relative concentration of confined carriers $(f)$ and the geometrical factor $\xi$ are considered as global (i.e., a single value of each one is assumed throughout all the fits of spectra with variable pump-probe delay, variable pump fluence and in equilibrium conditions). Thus, the entire series of conductivity and photoconductivity measurements for a particular sample has a shared set of these material parameters. The vital parameter, which is allowed to vary among all the spectra, is the carrier temperature, $T_{c}$; clearly, this quantity should vary with time and with the pump fluence. The Drude amplitude of the species with parabolic bands $\sigma_{D}^{B, 0}$ scales linearly with the pump fluence; however, it is allowed to vary freely with the pumpprobe delay. The importance of this term is found to be only marginal. First, we will comment 
on the values of shared material parameters and, subsequently, we will show and analyze particular sets of spectra of the global fit.

\subsection{Global Parameters}

Table 2 summarizes the shared set of material parameters of samples MEG and QFSLG. For QFSLG, we obtain the Fermi energy $E_{F} \approx 390 \mathrm{meV}$ and the carrier mobility $\eta_{c} \approx$ $1140 \mathrm{~cm}^{2} \mathrm{~V}^{-1} \mathrm{~s}^{-1}$, a reasonable set of values for hydrogen intercalated graphene on the $\mathrm{SiC}$ substrate. Although our experiments cannot decide about the polarity of the doping, these results (along with the directly observed high concentration of mobile charges in steady-state conductivity spectra, see fits in Figure 1 obtained with the same set of parameters) are in line with the prediction of high p-doping and Fermi energy level in QFSLG samples due to the spontaneous polarization in $\mathrm{SiC}$ substrate. ${ }^{[49,50]}$ Generally, the Fermi energy (carrier mobility) for graphene films grown on $\mathrm{C}$-face has a lower (higher) value than that on $\mathrm{Si}$-face ${ }^{[51,52]}$ and our THz conductivity data support such trends.

We observe the resonance frequency and the damping close to $\omega_{0} / 2 \pi \approx 1.6 \mathrm{THz}$ and $\Gamma / 2 \pi \approx$ 3.7 THz for QFSLG while the values for MEG are 1.25 THz and 3.4 THz, respectively, at room temperature. These values are consistent with the doping of graphene layers given by Fermi energies reported in Table 2 and with the previously reported values for $\mathrm{SiC}$ grown epitaxial graphene without any patterning. ${ }^{[29]}$ The fit values of $\xi$ provide an estimation of the size $d_{G}$ of confinement regions of the order of a few micrometers (for $w \sim \mathrm{nm}, \varepsilon_{W} \sim 1$ ) both for MEG and for QFSLG.

Table 2. Fit values of shared (global) material parameters for samples MEG and QFSLG.

\begin{tabular}{|c|c|c|c|c|}
\hline Sample & $\begin{array}{c}\text { Fermi Energy } \\
E_{F}(\mathrm{meV})\end{array}$ & $\begin{array}{c}\text { Carrier mobility } \\
\eta_{c}\left(\mathrm{~cm}^{2} \mathrm{~V}^{-1} \mathrm{~s}^{-1}\right)\end{array}$ & $\xi\left(\mathrm{mm}^{-1}\right)$ & $f(\%)$ \\
\hline
\end{tabular}




\begin{tabular}{|c|c|c|c|c|}
\hline MEG & 180 & 2700 & 0.22 & 62 \\
\hline QFSLG & 390 & 1140 & 0.16 & 80 \\
\hline
\end{tabular}

Our experiments clearly indicate that the observed plasmonic photoconductivity is isotropic and that it exhibits a very similar spectral behavior on both sides of the substrate. Indeed, such plasmonic response seems to be inherent to epigraphene grown on $\mathrm{SiC}$ and the imprinted spatial confinement is the same for both substrate sides and its directional variation is not significant. This is in contrast with the existence of terraces on SiC surface bringing some structural anisotropy to the samples and with the fact that the graphene grown on C-face is usually reported to feature considerably more wrinkles and less uniformity due to its inhomogeneous character than the graphene grown on Si-face ${ }^{[53,54]}$. It then seems that these two features cannot represent the direct origin of the confinement. We can speculate that the confinement could be related to the dimension of single crystalline graphene flakes with perfect conductivity surrounded by dead layers with defects. The size of such flakes determined during the growth then could be related to some extent to the spacing of terraces.

\subsection{Evolution of photoconductivity spectra}

Figure 6 shows the fitted photoconductivity spectra for various pump fluences and at a delay of $\tau_{p} \approx 1.5-2.0 \mathrm{ps}$ after the pump-probe signal peak. The fits provide details on the evolution of the carrier temperatures with fluence variation. The absorbed pump fluence was varied over two orders of magnitude in the range $\phi=(0.16-16) \times 10^{12}$ photons $/ \mathrm{cm}^{2}$; the fitted carrier temperature increases to $1050 \mathrm{~K}$ for the highest pump fluence in QFSLG. In Figure 3 we show also the fits to experimental data in a broader spectral range at the photon fluence of $\phi=8 \times$ $10^{12}$ photons $/ \mathrm{cm}^{2}$ to demonstrate the consistency of our model with broadband spectra up to 
$12 \mathrm{THz}$. Apparently, the fits for QFSLG are better than those for MEG. This may be related to our effective monolayer approximation assumed for MEG, while QFSLG is a true monolayer.
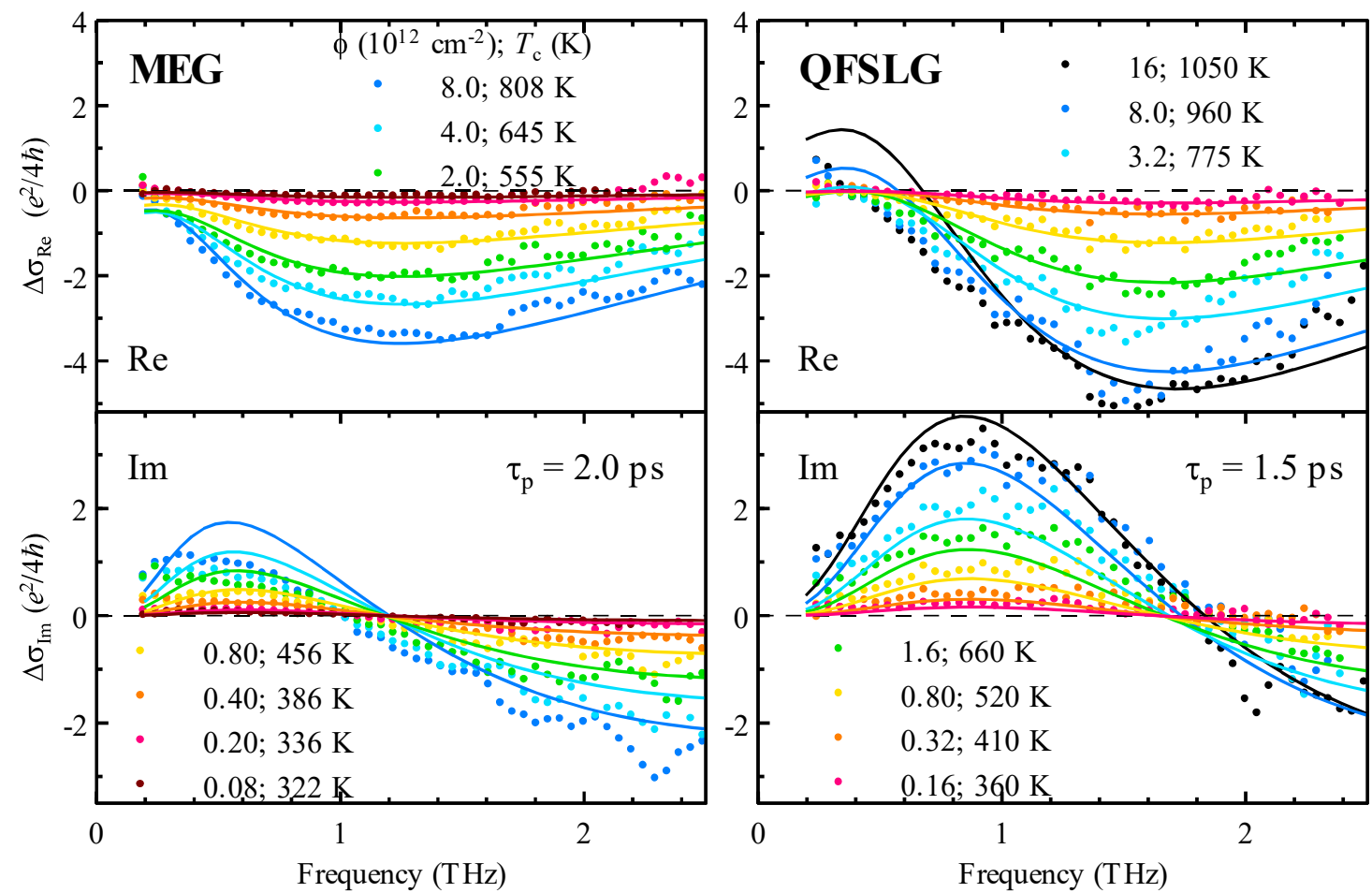

Figure 6. Transient sheet conductivity spectra of samples MEG and QFSLG for variable pump fluence $\phi$ and at a constant pump probe delay of $1.5 \mathrm{ps}(Q F S L G)$ and $2.0 \mathrm{ps}(M E G)$. The solid lines represent the global fit using (Equation 14). The carrier temperatures $T_{c}$ determined from the fit are indicated in the legends.

Figure 7 shows the evolution of the photoconductivity with pump-probe delay for samples MEG and QFSLG $\left(\phi=8 \times 10^{12}\right.$ photons $\left./ \mathrm{cm}^{2}\right)$. The spectra at delays beyond $4 \mathrm{ps}$ were found to contain a significant positive Drude contribution. Due to the positive Drude term and the carrier cooling, the spectrum of $\Delta \sigma_{R e}$ gradually increases and crosses the zero-conductivity line with increasing pump-probe delay. 

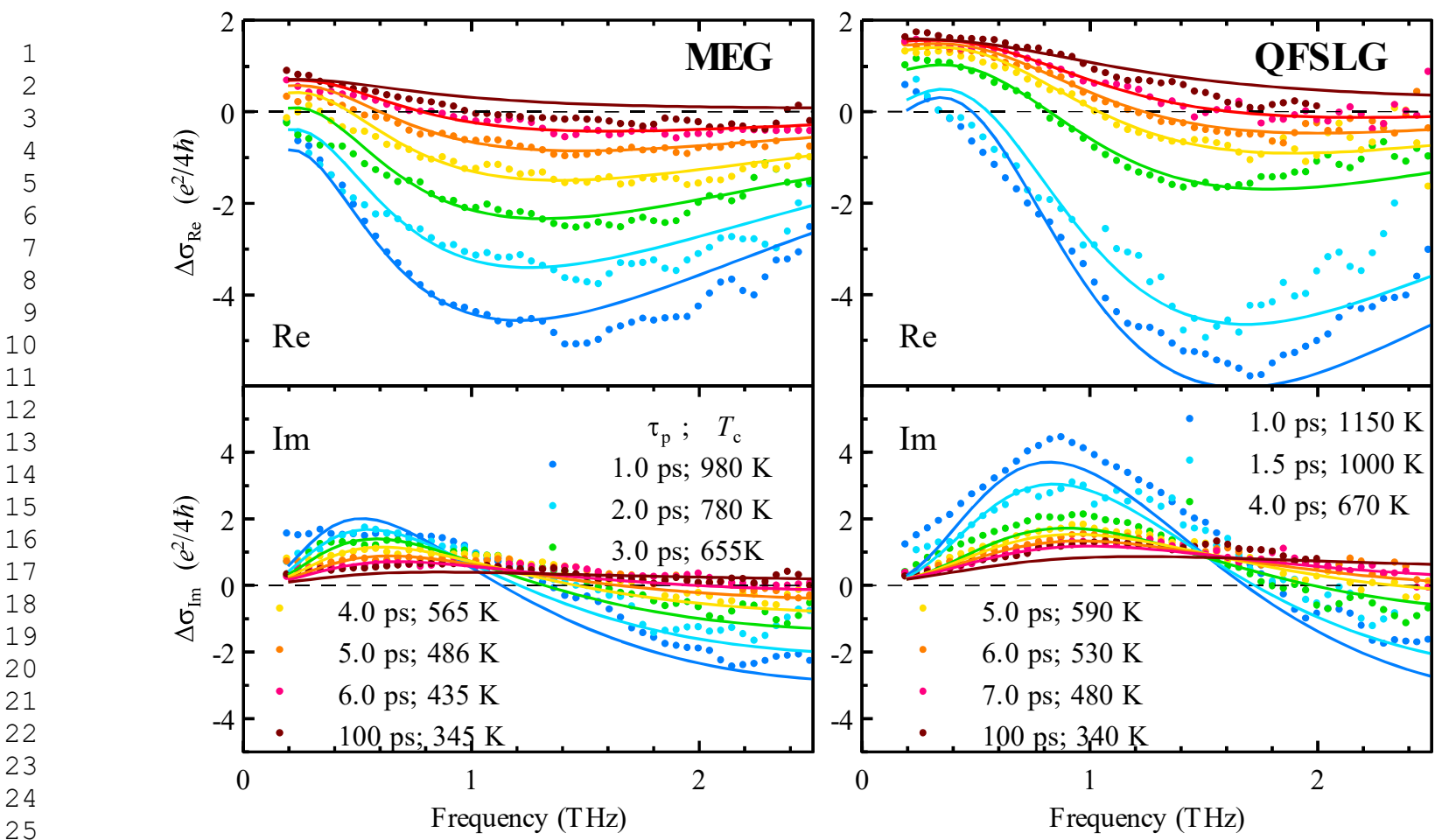

Figure 7. Complex photoconductivity spectra of samples MEG and QFSLG for variable pump-probe delay $\tau_{p}$ and at a constant absorbed photon fluence of $\phi=8 \times 10^{12} \mathrm{~cm}^{-2}$. The solid lines represent the global fit using (Equation 14). The pump-probe delay $\tau_{p}$ and the carrier temperature $T_{c}$ are indicated in the legends.
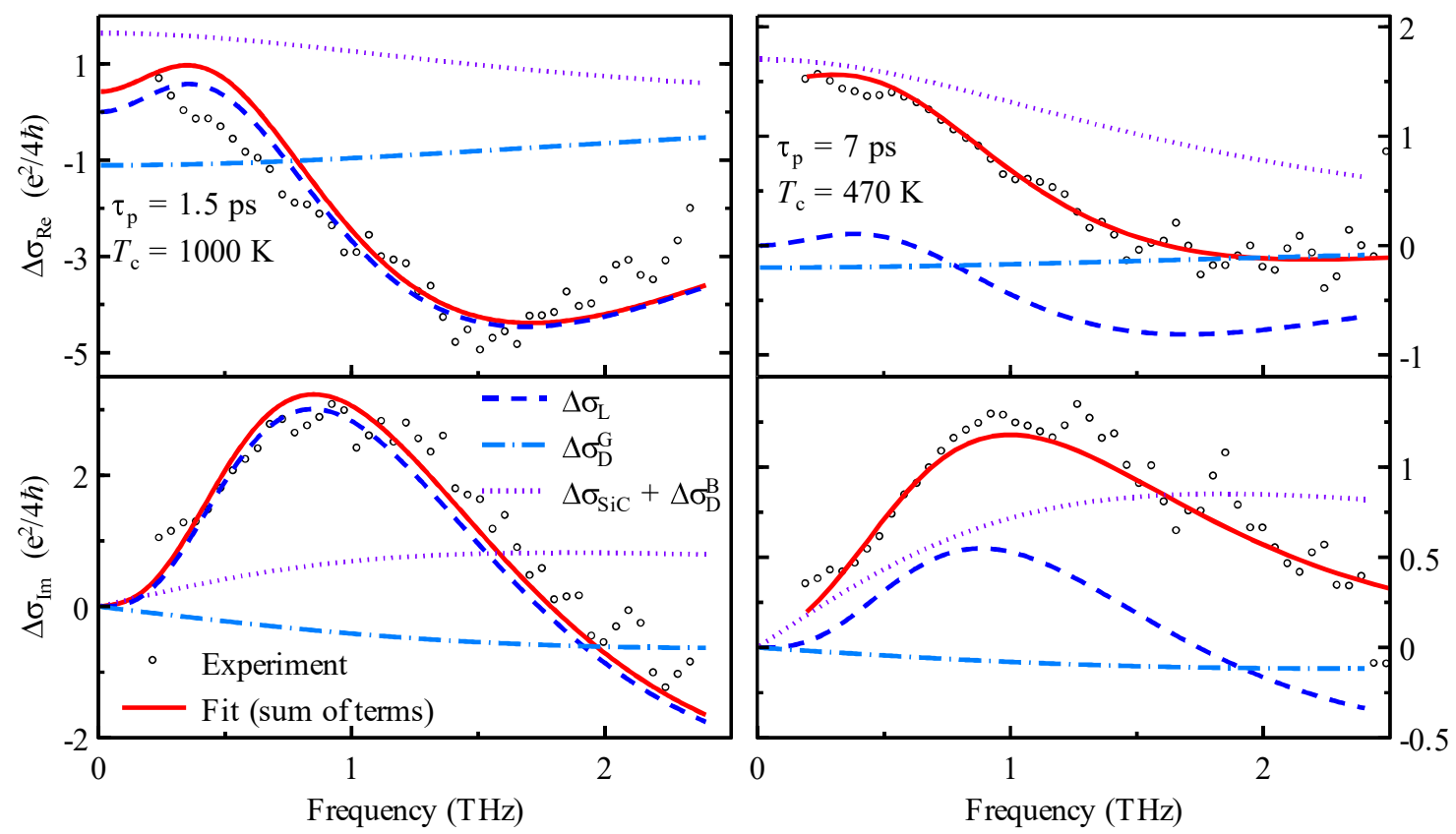

Figure 8. Decomposition of the fit of time resolved sheet conductivity spectra (QFSLG, $\phi=8 \times$ $10^{12} \mathrm{~cm}^{-2}$ ) into individual components of the model (Equation 14) as explained in the legend. Left panel: spectra at 1.5 ps after photoexcitation; the Lorentz contribution of localized carriers dominates. The Drude term of graphene partially compensates the Drude terms due to carriers in parabolic-band 
species (SiC and possibly other inhomogeneities in graphene layers) in agreement with Figure 2(a). Right panel: spectrum at 7 ps after photoexcitation; at the corresponding lower carrier temperatures the overall graphene contribution progressively becomes less pronounced than other Drude components.

The early signals and their fits decomposed into individual components are also shown in Figure 8. We can observe here an initial dominant contribution of the graphene terms due to the elevated carrier temperature (left panel of Figure 8); the shape of these spectra is clearly given by the Lorentz contribution of confined carriers. At moderate pump-probe delays (right panel), the carrier temperature is significantly closer to the lattice temperature and the Drude contribution of parabolic-band species (decaying on the sub-ns scale) starts to dominate over the graphene conductivity; the spectra thus start to approach the standard Drude-like shape.

\subsection{Carrier Cooling}

The initial carrier temperature in graphene layers reached upon photoexcitation is shown in Figure 9; the time evolution of the carrier temperature $T_{c}$ for a particular pump fluence is then plotted in Figure 10(a). As observed, $T_{c}$ relaxes towards the lattice temperature $T_{l}$ very quickly in a few picoseconds after excitation; subsequently, the cooling considerably slows down.

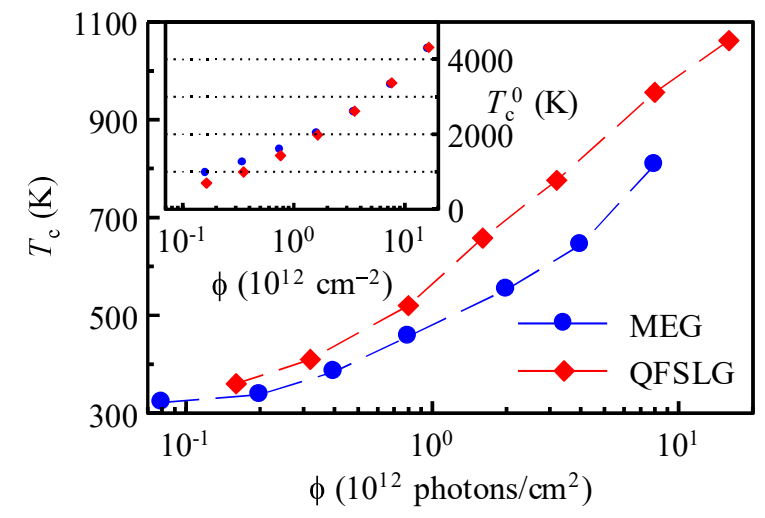

Figure 9. Variation of the carrier temperature $T_{c}$ versus absorbed photon fluence $\phi$ in samples $M E G$ $\left(\tau_{p} \approx 2 p s\right)$ and $Q F S L G\left(\tau_{p} \approx 1.5\right.$ ps). The inset shows the maximum possible value $T_{c}^{0}$ of the initial carrier temperature obtained from the energy conservation expressed by Equations (10) and (11) assuming the electronic subsystem retains the full energy deposited by the optical excitation. 
To obtain a deeper semi-quantitative insight let us consider the simplest approximation, in which the lattice retains all the energy lost from the electronic system during its cooling. For QFSLG, at $\phi=8 \times 10^{12}$ photons $/ \mathrm{cm}^{2}$ (corresponding to an absorbed fluence of $2 \mu \mathrm{J} / \mathrm{cm}^{2}$ ), the maximum carrier temperature $T_{c}^{0}$ of about $3400 \mathrm{~K}$ is first reached (see inset of Figure 9) and it drops to $1050 \mathrm{~K}$ within $1-2$ ps due to phonon mediated processes. Based on the energy conservation condition (Equation 10), about $94 \%$ of the energy deposited by the optical pulse is transferred from the electronic to the lattice system during this initial cooling phase. Assuming graphene's heat capacity follows a phonon dominated temperature dependence $C_{p h} \approx \alpha_{p} T_{l}$ with $\alpha_{p} \approx 1.71 \mu \mathrm{J} \mathrm{m}^{-2} \mathrm{~K}^{-2},{ }^{[55]}$ the lattice would be heated to $T_{l} \approx 335 \mathrm{~K}$ by the first (i.e. direct) pump pulse due to this energy transfer. Note, however, that the subsequent etalon reflection pulses $(R \approx 20 \%)$ can lead to a further increase of the lattice temperature to $T_{l} \approx 343 \mathrm{~K}$ within the next $10-20 \mathrm{ps}$.
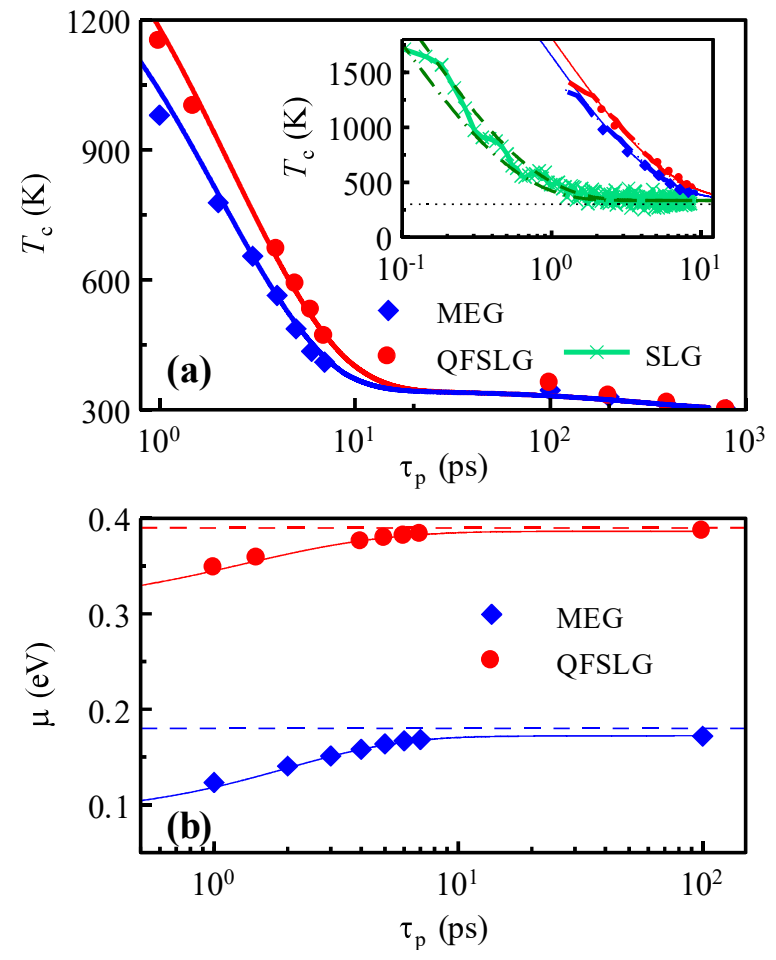

Figure 10. (a) Evolution of the carrier temperature $T_{c}$ after photoexcitation (absorbed photon fluence $\phi=8 \times 10^{12}$ photons $/ \mathrm{cm}^{2}$ ). Solid lines: fit by a combination of SCC model (Equation 12) and lattice cooling (Equation 13). Inset: comparison of linearly transformed $\Delta E\left(\tau_{p}\right)$ scan from Figure 2(a) 
(crosses) with the results of SCC model (lines). For SLG the experimental curve can be delimited by $S C C$ model prediction for $\beta / \alpha_{e}=1.8 \mathrm{~K}^{-1} \mathrm{~ns}^{-1}$ (upper) and $2.4 \mathrm{~K}^{-1} \mathrm{~ns}^{-1}$ (lower). (b) Evolution of the chemical potential $\mu\left(\tau_{p}\right)$ after photoexcitation; dashed lines show its equilibrium value (Fermi energy).

The underlying physical processes are thus as follows. The carriers transfer significant amount of their energy to the lattice in the rapid initial cooling phase ( $\lesssim 1 \mathrm{ps})$. The lattice heats up during this phase and no subsequent significant increase in its temperature can occur due to the higher lattice-to-electronic specific heat ratio of graphene. $T_{c}$ relaxes rapidly at the early instances after photoexcitation $\left(\lesssim 5 \mathrm{ps}\right.$ ) due to its large disparity with $T_{l}$. The cooling of electronic sub-system then dramatically slows down as $T_{c}$ approaches $T_{l}$ and the dynamics is described by a regime of very slow relaxation mostly governed by the cooling of graphene lattice.

We set up our fitting model by imposing $T_{l}\left(\tau_{p} \approx 1 \mathrm{ps}\right) \approx 335 \mathrm{~K}$ and $T_{l}\left(\tau_{\mathrm{p}} \approx 10 \mathrm{ps}\right) \approx 343 \mathrm{~K}$ (etalon excitation). For both MEG and QFSLG, the experimental data represented by $T_{c}\left(\tau_{p}\right)$ agree well with a combination of SCC model (12) and lattice cooling (13), see fits in Figure 10. The lattice cooling time constant is $\tau_{l} \approx 300$ ps for both samples. The fit values of $\beta / \alpha_{e}$ for QFSLG and MLG are $0.25 \mathrm{~K}^{-1} \mathrm{~ns}^{-1}$ and $0.30 \mathrm{~K}^{-1} \mathrm{~ns}^{-1}$, respectively. In order to estimate the disorder mean free path we need to establish the value of the acoustic deformation potential $V_{D}$. Its value is expected between 10 and $30 \mathrm{eV}$ and it was experimentally determined from temperature dependent resistivity as $V_{D} \approx 29 \mathrm{eV}$ for suspended graphene, ${ }^{[56]}$ and in the range $V_{D} \approx 17-21 \mathrm{eV}$ for the unsuspended graphene. ${ }^{[57,58]}$ Assuming here $V_{D}=20 \mathrm{eV}$ we find $l_{\mathrm{dis}} \approx 9 \mathrm{~nm}$ and $\approx 7.5 \mathrm{~nm}$ for QFSLG and MGL, respectively.

In order to provide a rough estimate also for the sample SLG, we performed the following analysis. We compared the curves representing the raw pump probe scans in Figure 2(a) for QFSLG and MEG with the $T_{c}$ dependences shown in Figure 10(a): for this purpose, we linearly transformed their vertical scale, i.e., $\Delta E$ to $T_{c}$ such that $T_{c}\left(\tau_{p}=0\right)=3400 \mathrm{~K}$. The curves show a very reasonable agreement. We then performed the same transformation for the pump-probe 
scan of SLG. The results are shown in the inset of Figure 10(a); the SCC modeling delimits the experimental curve for $\beta / \alpha_{\mathrm{e}}=1.8$ and $2.4 \mathrm{~K}^{-1} \mathrm{~ns}^{-1}$; thus, for SLG the degree of disorder is significantly higher and the disorder mean free path might be as short as $1 \mathrm{~nm}$. It is reasonable to assume a similar degree of disorder in QFBLG (though not thoroughly analyzed), considering the short positive dynamics observed in the sample in Figure 2(a).

The described phenomena can be also represented within the framework of the chemical potential. As observed in Figure $10(\mathrm{~b}), \mu\left(\tau_{p}\right)$ initially drops from its equilibrium value $E_{F}$ due to the carrier redistribution in the conduction band $\sim 1$ ps after photoexcitation; the decrease of the chemical potential values then leads to the observed induced transparency in the differential signal. Values close to equilibrium are recovered after a few picoseconds.

We thus come to the conclusion that, in epigraphene grown both on $\mathrm{C}$ - and $\mathrm{Si}$-side of $\mathrm{SiC}$ substrate, the ultrafast dynamics is entirely governed by the time-dependent carrier temperature (or, equivalently, the chemical potential). The temperature or chemical potential are inherently nonlinear parameters in the carrier response. Recently, the carrier temperature has been shown to play the key role for strong THz nonlinear conductivity response in CVD graphene. ${ }^{[6]}$ The paper demonstrates odd-harmonics generation in the $\mathrm{THz}$ range in graphene driven by intense $\sim 20$ ps long pulses centered at $0.3 \mathrm{THz}$ generated at TELBE facility. Our studies complement these conclusions with the finding that the temperature relaxation governing the carrier response occurs on $\mathrm{a} \lesssim 5$ ps time scale.

\section{Conclusion}

We determined steady-state conductivity and ultrafast photoconductivity spectra in the $\mathrm{THz}$ range of five allotropes of graphene epitaxially grown on $\mathrm{SiC}$ substrates. The spectra of graphene layers reveal a dominant localized carrier response on the picosecond time scale with an additional weaker free-carrier contribution. Observation of such plasmonic response is a 
direct proof of natural confinement potentials for carriers in epitaxially grown graphene. Our results clearly show that for both $\mathrm{C}$ - and $\mathrm{Si}$-side grown layers, the plasmonic carrier confinement in micrometer-sized graphene domains is the leading phenomenon determining the character of the $\mathrm{THz}$ response on a few picosecond timescale. This short-lived plasmonic response scales nonlinearly with initial photocarrier density and it is entirely governed by ultrafast carrier temperature relaxation.

A relatively simple two-temperature differential model (equilibrium carriers vs. optically heated carriers) complemented with a Drude term from substrate and other impurities was set up; the model explains the complex variations of the photoconductivity spectra across a wide range of pump fluences and pump-probe delays and accounts for the observed interplay between induced $\mathrm{THz}$ transparency and absorption in the samples. Simultaneous analyses of the steady-state $(\sigma)$ and transient $(\Delta \sigma)$ sheet conductivity spectra provide important electronic parameters such as Fermi energy, carrier mobility $\left(\approx 1100\right.$ and $2700 \mathrm{~cm}^{2} \mathrm{~V}^{-1} \mathrm{~s}^{-1}$ for QFSLG and MEG, respectively) and confinement length (of the order of a few $\mu \mathrm{m}$ ). The relative strength of the plasmonic (Lorentz term, 80 and 60\% for QFSLG and MEG, respectively) and free carrier (Drude term in graphene and in other species) conductivity provides a quantitative picture of the extent of inhomogeneity in the graphene allotrope and the presence of remnant species with parabolic bands. In addition, the transient photoconductivity spectra enable us to understand the ultrafast thermalization dynamics and to estimate the disorder mean free path $(\sim 7-9 \mathrm{~nm})$ of carriers in QFSLG and MEG. The initial carrier cooling is governed by the super-collision process lasting a few picoseconds; the subsequent dynamics is dictated by a slow transfer of graphene lattice heat to the substrate via acoustic phonon emission. 


\section{Materials and methods}

Samples

We used the $6 \mathrm{H}-\mathrm{SiC}$ on-axis, a semi-insulating substrate (II-VI Inc). We diced the substrate into $5 \times 5 \mathrm{~mm}^{2}$ squares, cleaned them in acetone and isopropanol, and we annealed them in argon at 1050 mbar, and flow 8-10 slph as a graphene growth ambient conditions. The growth conditions and nominal properties of the prepared samples are summarized in Table 1. One sample is MEG on a C-face. The four other samples were grown on Si face: BL, SLG, and two intercalated samples (QFSLG and QFBLG), which are formed from BL and SLG after intercalation. We performed the intercalation in a palladium cell purified ambient at 1050 mbar, hydrogen flow $3.0 \mathrm{slph}$, and gradually lowered temperature from 1120 to $500^{\circ} \mathrm{C}$. The cooling rate was below $1{ }^{\circ} \mathrm{C} / \mathrm{s}$ for the intercalation procedure. Further details on growth can be found in J. Kunc et al. ${ }^{[59,60]}$ In Table 1 we indicate the number of layers covering most of the samples' area. However, small islands of \pm 1 layer do exist in all the samples, as discussed, e.g., in our previous work. ${ }^{[61]}$ An AFM image of sample QFSLG, Raman spectra and maps of all the samples and results of magneto-optical FTIR measurements are shown in the Supporting material (Sections 7 and 8).

\section{THz steady-state spectroscopy}

The THz steady-state conductivity spectra of our samples were measured on transmission using a conventional femtosecond oscillator based time-domain $\mathrm{THz}$ spectroscopy setup basically described in P. Kužel et al. ${ }^{[62]}$ The sheet conductivity of a 2D material on substrate was calculated from the Tinkham formula generalized for phase-sensitive THz measurements as described in the Supporting material (Sec. 3). A special care has been taken to optimize the respective thicknesses of the substrate with film and reference substrate ${ }^{[63,64]}$, see also 
Supporting material, Sec. 3. This approach allows for a valuable determination of the complex sheet conductivity $\sigma(\omega)$ for very thin weakly conducting films like our graphene samples.

\section{Transient THz spectroscopy}

The photoconductivity of the studied samples was measured at room temperature by means of a conventional optical pump $-\mathrm{THz}$ probe setup ${ }^{[65]}$ driven by Ti:sapphire ultrafast amplified laser system (Spitfire ACE, $5 \mathrm{kHz}$ repetition rate, 40 fs pulse length), see Supporting material (Sec. 4) for more details. In this paper we characterize systematically the conditions of photoexcitation at $800 \mathrm{~nm}$ by the density of absorbed photons (photon fluence) $\phi$; this parameter was varied by about two orders of magnitude in our experiments.

A part of the experiments was performed using an optical pump - multi-THz probe scheme reaching frequencies up to $20 \mathrm{THz}$ with the help of a custom-built spectrometer utilizing twocolor mixing in plasma ${ }^{[66]}$ and air-biased coherent detection $(\mathrm{ABCD})$ scheme, ${ }^{[67]}$ see Supporting material (Sec. 5) for the setup description.

Two kinds of measurements were performed.

(i) The pump-probe scans were obtained if the gated time-domain detection was set into the position of the maximum of the transient wave form $\Delta E_{\max }$ and the pump-probe delay $\tau_{p}$ was scanned to obtain $\Delta E_{\max }\left(\tau_{p}\right) / E_{0}$.

(ii) The transient THz wave forms $\Delta E(t)$ were measured for particular pump-probe delays $\tau_{p}$ and pump fluences $\phi$. The sheet photoconductivity spectra were then obtained using: ${ }^{[68]}$

$$
\Delta \sigma\left(\omega ; \tau_{p}, \phi\right)=-\frac{1+N_{s}}{z_{0}} \frac{\Delta E(\omega)}{E_{0}(\omega)}
$$

where $N_{s}(\approx 3.14)$ is the $\mathrm{THz}$ refractive index of $\mathrm{SiC}$ and $z_{0}$ is the vacuum wave impedance. Note that the term of "conductivity" is often used in the relevant literature as a convenient (but 
somewhat ambiguous) abbreviation of the sheet conductivity. Also, in this paper, by simply writing a "(photo)conductivity" we always mean the sheet (photo)conductivity of the studied 2D system.

\section{Supporting Information:}

Supporting Information is available from the Wiley Online Library or from the author.

\section{Acknowledgment:}

This work was supported by the Operational Programme "Research, Development and Education" financed by European Structural and Investment Funds and the Czech Ministry of Education, Youth and Sports (Project No. SOLID21-CZ.02.1.01/0.0/0.0/16_019/0000760) and by the Czech Science Foundation (Project No. 19-28375X). CzechNanoLab project LM2018110 funded by MEYS CR is gratefully acknowledged for the financial support of the sample fabrication at CEITEC Nano Research Infrastructure. JK wish to thank Czech Science Foundation (project No. 19-12052S) for the kind support. We also acknowledge the support of the LNCMI-CNRS in Grenoble, a member of the European Magnetic Field Laboratory (EMFL).

\section{Conflict of Interests:}

The authors declare no conflict of interest.

\section{References}

[1] Y. M. Lin, C. Dimitrakopoulos, K. A. Jenkins, D. B. Farmer, H. Y. Chiu, A. Grill, P. Avouris, Science. 2010, 327, 662.

[2] M. Mittendorff, S. Winnerl, J. Kamann, J. Eroms, D. Weiss, H. Schneider, M. Helm, Appl. Phys. Lett. 2013, 103, 021113. 
[3] A. Y. Zhu, E. Cubukcu, 2D Mater. 2015, 2, 032005.

[4] S. Bae, H. Kim, Y. Lee, X. Xu, J. S. Park, Y. Zheng, J. Balakrishnan, T. Lei, H. Ri Kim, Y. Il Song, Y. J. Kim, K. S. Kim, B. Özyilmaz, J. H. Ahn, B. H. Hong, S. Iijima, Nat. Nanotechnol. 2010, 5, 574.

[5] H. Medina, Y. C. Lin, C. Jin, C. C. Lu, C. H. Yeh, K. P. Huang, K. Suenaga, J. Robertson, P. W. Chiu, Adv. Funct. Mater. 2012, 22, 2123.

[6] H. A. Hafez, S. Kovalev, J. C. Deinert, Z. Mics, B. Green, N. Awari, M. Chen, S. Germanskiy, U. Lehnert, J. Teichert, Z. Wang, K. J. Tielrooij, Z. Liu, Z. Chen, A. Narita, K. Müllen, M. Bonn, M. Gensch, D. Turchinovich, Nature 2018, 561, 507.

[7] P. A. Obraztsov, M. G. Rybin, A. V. Tyurnina, S. V. Garnov, E. D. Obraztsova, A. N. Obraztsov, Y. P. Svirko, Nano Lett. 2011, 11, 1540.

[8] J. Christensen, A. Manjavacas, S. Thongrattanasiri, F. H. L. Koppens, F. J. García De Abajo, ACS Nano 2012, 6, 431.

[9] M. S. Ukhtary, R. Saito, Carbon. 2020, 167, 455.

[10] T. Low, P. Avouris, ACS Nano 2014, 8, 1086.

[11] L. Wang, C. Liu, X. Chen, J. Zhou, W. Hu, X. Wang, J. Li, W. Tang, A. Yu, S. W. Wang, W. Lu, Adv. Funct. Mater. 2017, 27.

[12] M. M. Jadidi, K. M. Daniels, R. L. Myers-Ward, D. K. Gaskill, J. C. König-Otto, S. Winnerl, A. B. Sushkov, H. D. Drew, T. E. Murphy, M. Mittendorff, ACS Photonics 2019, 6, 302.

[13] E. V. Iski, E. N. Yitamben, L. Gao, N. P. Guisinger, Adv. Funct. Mater. 2013, 23, 2554.

[14] C. Berger, Z. Song, T. Li, X. Li, A. Y. Ogbazghi, R. Feng, Z. Dai, N. Alexei, M. E. H. Conrad, P. N. First, W. A. De Heer, J. Phys. Chem. B 2004, 108, 19912. 
[15] S. N. Yannopoulos, A. Siokou, N. K. Nasikas, V. Dracopoulos, F. Ravani, G. N. Papatheodorou, Adv. Funct. Mater. 2012, 22, 113.

[16] C. Riedl, C. Coletti, T. Iwasaki, A. A. Zakharov, U. Starke, Phys. Rev. Lett. 2009, 103, 246804.

[17] J. Hass, F. Varchon, J. E. Millán-Otoya, M. Sprinkle, N. Sharma, W. A. De Heer, C. Berger, P. N. First, L. Magaud, E. H. Conrad, Phys. Rev. Lett. 2008, 100, 125504.

[18] J. D. Emery, V. H. Wheeler, J. E. Johns, M. E. McBriarty, B. Detlefs, M. C. Hersam, D. Kurt Gaskill, M. J. Bedzyk, Appl. Phys. Lett. 2014, 105, 161602.

[19] F. Speck, J. Jobst, F. Fromm, M. Ostler, D. Waldmann, M. Hundhausen, H. B. Weber, T. Seyller, Appl. Phys. Lett. 2011, 99, 122106.

[20] C. Yu, Q. Liu, J. Li, W. Lu, Z. He, S. Cai, Z. Feng, Appl. Phys. Lett. 2014, 105, 183105.

[21] R. Ulbricht, E. Hendry, J. Shan, T. F. Heinz, M. Bonn, Rev. Mod. Phys. 2011, 83, 543.

[22] P. Kužel, H. Němec, Adv. Opt. Mater. 2020, 8, 1900623.

[23] S. Kar, V. L. Nguyen, D. R. Mohapatra, Y. H. Lee, A. K. Sood, ACS Nano 2018, 12, 1785.

[24] H. Yan, F. Xia, W. Zhu, M. Freitag, C. Dimitrakopoulos, A. A. Bol, G. Tulevski, P. Avouris, ACS Nano 2011, 5, 9854.

[25] S. Kar, S. Jayanthi, E. Freysz, A. K. Sood, Carbon. 2014, 80, 762.

[26] J. Kim, J. Oh, C. In, Y. S. Lee, T. B. Norris, S. C. Jun, H. Choi, ACS Nano 2014, 8, 2486.

[27] S. Kar, Y. Su, R. R. Nair, A. K. Sood, ACS Nano 2015, 9, 12004.

[28] M. M. Jadidi, J. C. König-Otto, S. Winnerl, A. B. Sushkov, H. D. Drew, T. E. Murphy, 
M. Mittendorff, Nano Lett. 2016, 16, 2734.

[29] I. Crassee, M. Orlita, M. Potemski, A. L. Walter, M. Ostler, T. Seyller, I. Gaponenko, J. Chen, A. B. Kuzmenko, Nano Lett. 2012, 12, 2470.

[30] H. Němec, F. Kadlec, P. Kužel, J. Chem. Phys. 2002, 117, 8454.

[31] H. Němec, F. Kadlec, S. Surendran, P. Kužel, P. Jungwirth, J. Chem. Phys. 2005, 122, 104503.

[32] C. J. Docherty, C. Te Lin, H. J. Joyce, R. J. Nicholas, L. M. Herz, L. J. Li, M. B. Johnston, Nat. Commun. 2012, 3, 1226.

[33] M. T. Mihnev, F. Kadi, C. J. Divin, T. Winzer, S. Lee, C. H. Liu, Z. Zhong, C. Berger, W. A. De Heer, E. Malic, A. Knorr, T. B. Norris, Nat. Commun. 2016, 7, 11617.

[34] S. Kar, D. R. Mohapatra, A. K. Sood, Nanoscale 2018, 10, 14321.

[35] S. Kar, D. R. Mohapatra, E. Freysz, A. K. Sood, Phys. Rev. B 2014, 90, 165420.

[36] H. A. Hafez, X. Chai, Y. Sekine, M. Takamura, K. Oguri, I. Al-Naib, M. M. Dignam, H. Hibino, T. Ozaki, Phys. Rev. B 2017, 95, 165428.

[37] A. J. Frenzel, C. H. Lui, Y. C. Shin, J. Kong, N. Gedik, Phys. Rev. Lett. 2014, 113, 056602.

[38] Z. Fei, A. S. Rodin, W. Gannett, S. Dai, W. Regan, M. Wagner, M. K. Liu, A. S. McLeod, G. Dominguez, M. Thiemens, A. H. Castro Neto, F. Keilmann, A. Zettl, R. Hillenbrand, M. M. Fogler, D. N. Basov, Nat. Nanotechnol. 2013, 8, 821.

[39] J. Chen, M. L. Nesterov, A. Y. Nikitin, S. Thongrattanasiri, P. Alonso-González, T. M. Slipchenko, F. Speck, M. Ostler, T. Seyller, I. Crassee, F. H. L. Koppens, L. MartinMoreno, F. J. García De Abajo, A. B. Kuzmenko, R. Hillenbrand, Nano Lett. 2013, 13, 6210. 
[40] L. Ju, B. Geng, J. Horng, C. Girit, M. Martin, Z. Hao, H. A. Bechtel, X. Liang, A. Zettl, Y. R. Shen, F. Wang, Nat. Nanotechnol. 2011, 6, 630.

[41] E. H. Hwang, S. Das Sarma, Phys. Rev. B 2008, 77, 115449.

[42] T. Stauber, N. M. R. Peres, F. Guinea, Phys. Rev. B 2007, 76, 205423.

[43] I. Rychetský, D. Nuzhnyy, J. Petzelt, Ferroelectrics 2020, 569, 9.

[44] D. Sun, C. Divin, C. Berger, W. A. De Heer, P. N. First, T. B. Norris, Phys. Rev. Lett. 2010, 104, 136802.

[45] P. A. George, J. Strait, J. Dawlaty, S. Shivaraman, M. Chandrashekhar, F. Rana, M. G. Spencer, Nano Lett. 2008, 8, 4248.

[46] E. H. Hwang, S. Das Sarma, Phys. Rev. B 2009, 79, 165404.

[47] H. Choi, F. Borondics, D. A. Siegel, S. Y. Zhou, M. C. Martin, A. Lanzara, R. A. Kaindl, Appl. Phys. Lett. 2009, 94, 172102.

[48] J. C. W. Song, M. Y. Reizer, L. S. Levitov, Phys. Rev. Lett. 2012, 109, 106602.

[49] J. Ristein, S. Mammadov, T. Seyller, Phys. Rev. Lett. 2012, 108, 246104.

[50] D. Momeni Pakdehi, P. Schädlich, T. T. N. Nguyen, A. A. Zakharov, S. Wundrack, E. Najafidehaghani, F. Speck, K. Pierz, T. Seyller, C. Tegenkamp, H. W. Schumacher, Adv. Funct. Mater. 2020, 30, 1.

[51] D. A. Siegel, C. G. Hwang, A. V. Fedorov, A. Lanzara, Phys. Rev. B 2010, 81, 241417(R).

[52] J. L. Tedesco, B. L. Vanmil, R. L. Myers-Ward, J. M. McCrate, S. A. Kitt, P. M. Campbell, G. G. Jernigan, J. C. Culbertson, C. R. Eddy, D. K. Gaskill, Appl. Phys. Lett. 2009, 95, 122102. 
[53] R. Pearce, X. Tan, R. Wang, T. Patel, J. Gallop, A. Pollard, R. Yakimova, L. Hao, Surf. Topogr. Metrol. Prop. 2015, 3, 015001.

[54] Luxmi, N. Srivastava, G. He, R. M. Feenstra, P. J. Fisher, Phys. Rev. B 2010, 82, 235406.

[55] E. Pop, V. Varshney, A. K. Roy, MRS Bull. 2012, 37, 1273.

[56] K. I. Bolotin, K. J. Sikes, J. Hone, H. L. Stormer, P. Kim, Phys. Rev. Lett. 2008, 101, 096802.

[57] J. H. Chen, C. Jang, S. Xiao, M. Ishigami, M. S. Fuhrer, Nat. Nanotechnol. 2008, 3, 206.

[58] C. R. Dean, A. F. Young, I. Meric, C. Lee, L. Wang, S. Sorgenfrei, K. Watanabe, T. Taniguchi, P. Kim, K. L. Shepard, J. Hone, Nat. Nanotechnol. 2010, 5, 722.

[59] J. Kunc, M. Rejhon, E. Belas, V. Dědič, P. Moravec, J. Franc, Phys. Rev. Appl. 2017, 8, 044011.

[60] J. Kunc, M. Rejhon, P. Hlídek, AIP Adv. 2018, 8, 045015.

[61] M. Rejhon, J. Kunc, J. Raman Spectrosc. 2019, 50, 465.

[62] P. Kužel, H. Němec, F. Kadlec, C. Kadlec, Opt. Express 2010, 18, 15338.

[63] C. Kadlec, F. Kadlec, H. Němec, P. Kužel, J. Schubert, G. Panaitov, J. Phys. Condens. Matter 2009, 21, 115902.

[64] K. L. Krewer, Z. Mics, J. Arabski, G. Schmerber, E. Beaurepaire, M. Bonn, D. Turchinovich, Opt. Lett. 2018, 43, 447.

[65] L. Fekete, P. Kužel, H. Němec, F. Kadlec, A. Dejneka, J. Stuchlík, A. Fejfar, Phys. Rev. $B$ 2009, 79, 115306.

[66] M. D. Thomson, M. Kreß, T. Löffler, H. G. Roskos, Laser Photonics Rev. 2007, 1, 349.

[67] N. Karpowicz, J. Dai, X. Lu, Y. Chen, M. Yamaguchi, H. Zhao, X. C. Zhang, L. Zhang, 
C. Zhang, M. Price-Gallagher, C. Fletcher, O. Mamer, A. Lesimple, K. Johnson, Appl. Phys. Lett. 2008, 92, 011131.

[68] H. K. Nienhuys, V. Sundström, Phys. Rev. B 2005, 71, 235110.

\section{TOC text}

The graphene electronics relies on various routes towards Fermi level tuning. We assess the plasmonic response of laser heated carriers on picosecond timescale in various allotropes of epitaxial graphene. This reveals ultrafast dynamics of the carriers' Fermi level and temperature, and their relaxation is mediated by disorder-assisted super collision cooling.

TOC figure $(55 \times 50)$

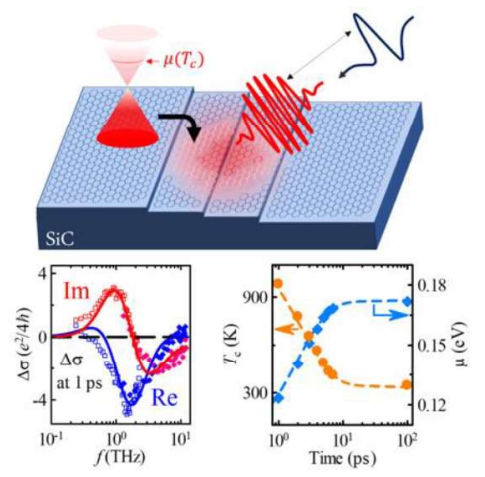

